\title{
Amniocentesis precoz y biopsia de vellosidad corial. Pérdidas fetales y anomalías congénitas en un grupo de gestantes brasileñas
}

\author{
Early amniocentesis and chorionic villus biopsy. Fetal losses and \\ congenital anomalies in a group of Brazilian pregnant women
}

Título abreviado: Amniocentesis precoz y biopsia de vellosidad corial

Luz Mery Bernal', María Consuelo Bernal², Thomaz Gollop ${ }^{3}$

\section{Resumen}

En el Instituto de Medicina Fetal y Genética Humana de São Paulo se ofrecen, a las gestantes que tienen un riesgo aumentado para anomalías cromosómicas, diferentes técnicas, entre ellas, la Biopsia de Vellosidad Corial Transabdominal (BVCTA) y la Amniocentesis Precoz (AP). El objetivo del presente estudio es realizar una comparación de la frecuencia de pérdidas fetales y anomalías congénitas presentadas en cada uno de los procedimientos, ambos realizados por los mismos operadores, en la misma edad gestacional (12-14 6/7 semanas) y bajo un abordaje transabdominal. Fueron analizadas retrospectivamente $432 \mathrm{AP}$ y 418 BVCTA. Todos los procedimientos de colecta fueron monitorizados por ultrasonografía. La frecuencia de pérdidas fetales espontáneas fue del 4,9\% en AP y de 5,3\% en BVCTA, una diferencia no significativa. No se encontraron diferencias significativas entre los dos procedimientos al comparar las frecuencias de pérdidas en cada semana de gestación. Sangrado y pérdida de líquido amniótico fueron más frecuentes en AP que en la BVCTA. Esa diferencia fue significativa en el caso de la pérdida de líquido amniótico. En algunos casos este hallazgo se relacionó con pérdida fetal. La incidencia de prematuridad y bajo peso al nacimiento no difirió significativamente entre los dos procedimientos. La mayor frecuencia de problemas respiratorios registrada en AP no fue significativa en comparación con BVCTA. No se observó diferencia significativa en la incidencia de anomalías músculo-esqueléticas. La amniocentesis después de catorce semanas presenta un bajo riesgo de pérdida fetal o anomalías congénitas. La BVCTA, debe ser realizada alrededor de la semana doce de gestación.

Palabras claves: amniocentesis precoz, biopsia de vellosidad corial, pérdida fetal, anomalías congénitas.

\section{Abstract}

In the Institute of Fetal Medicine and Human Genetics of São Paulo are offered, pregnant women have an increased risk for chromosomal abnormalities, different techniques, among them, Transabdominal Corial Vellosity Biopsy (BVCTA) and Precocious Amniocentesis (AP). The

\footnotetext{
Investigadora del Grupo Bioinnova. Escuela de Ciencias de la Salud. Universidad Nacional Abierta y a Distancia. Instituto de Medicina Fetal

y Genética Humana. Brasil.

2. Investigadora del Grupo Bioinnova. Escuela de Ciencias de la Salud. Universidad Nacional Abierta y a Distancia.

3. Investigador Universidad de Sao Paulo, Brasil. Instituto de Medicina Fetal y Genética Humana. Brasil.
} 
objective of this study is to compare the frequency of Hepatitis and congenital anomalies presented in all procedures, both performed by operators, in the same gestational age (12-14 6/7 weeks) and under a transabdominal approach. $432 \mathrm{AP}$ and 418 BVCTA were analyzed retrospectively. All collection procedures were monitored by ultrasonography. The spontaneous fetal frequency was 4.9\% in AP and 5.3\% in BVCTA, a non-significant difference. There is no difference in results compared to gestation times. Bleeding and loss of amniotic fluid were more frequent in AP than in BVCTA. That difference was significant in the case of the loss of amniotic fluid. In some cases, this finding was related to fetal loss. The incidence of prematurity and birth weight without difference between the two procedures. The highest frequency of respiratory problems recorded in AP was not significant compared to BVCTA. There is no significant difference in the incidence of musculoskeletal abnormalities. Amniocentesis after 14 weeks presents a low risk of fetal loss or congenital anomalies. The BVCTA should be close to the twelfth week of gestation.

Keywords: Early amniocentesis, chorionic villus biopsy, fetal loss, congenital anomalies.

\section{Introducción}

El Diagnóstico Prenatal (DPN) es un conjunto de técnicas destinadas a establecer un diagnóstico fetal aún en el periodo de vida intrauterina. Está dirigido principalmente a parejas con mayor riesgo de presentar un embarazo de un hijo con una anomalía genética o congénita. Su objetivo fundamental es lograr la identificación de anomalías cromosómicas, malformaciones, enfermedades metabólicas, mendelianas y otras alteraciones eventualmente adquiridas durante la gestación y con repercusiones sobre el feto. La Amniocentesis Tradicional (AT) fue el primer procedimiento invasivo utilizado para recolectar líquido amniótico destinado al análisis citogenético del feto. Se trata de una técnica relativamente simple cuya seguridad y confianza diagnóstica se encuentra bien establecida (1-6). Aunque sea considerado patrón, el procedimiento es limitado pues sólo puede realizarse en el segundo trimestre del embarazo y la edad gestacional en que se encuentra la paciente cuando los resultados son suministrados varía entre las semanas 17 y 18 (7). La introducción de los procedimientos de Biopsia de Vellosidad Corial (BVC) y más tarde de Amniocentesis Precoz (AP) se llevó a cabo por la demanda de diagnósticos prenatales en periodos menos tardíos del embarazo. La colecta de la BVC es realizada por vía transcervical (TC) entre la semana 10 y la sema- na 11 de gestación o por vía transabdominal (TA), generalmente a partir de la semana 11. La elección de la vía de colecta depende básicamente de la opción de la paciente, de las condiciones anatómicas de la placenta y de la experiencia del profesional encargado. La BVC-TC fue introducida en Brasil en 1985 por Gollop y otros autores; y la BVC-TA en 1988 por Montenegro y otros. Los primeros estudios relatando la experiencia brasilera fueron publicados poco tiempo después (8-10). La AP puede ser realizada a partir de la semana 12 de gestación; su seguridad ha sido defendida y cuestionada desde su establecimiento por las intercurrencias asociadas $(6,11-13)$.

En Brasil, la AP fue introducida en 1992 (14) y desde entonces ha sido rutinariamente ofrecida en el Instituto de Medicina Fetal y Genética Humana de Sao Paulo Brasil.

\section{Materiales y métodos}

En el Instituto de Medicina Fetal y Genética Humana de Sáo Paulo se ofrecen, entre otras técnicas, la Biopsia de Vellosidad Corial Transabdominal (BVCTA) y la Amniocentesis Precoz (AP) a las pa- 
cientes con riesgo aumentado para anomalías cromosómicas. Se realizó un análisis retrospectivo de un periodo de cuatro años, en una población de mujeres embarazadas que fueron atendidas por riesgo de portar un feto con anomalía cromosómica. De un total de 1.410 pacientes, 505 fueron sometidas a AP y 905 a BVCTA. Excluyéndose casos con gestaciones gemelares, así como procedimientos realizados fuera del periodo entre 12 0/7 y 14 y 6/7 semanas, se obtuvieron para análisis 432 AP y 418 AVC-TA.

Previo al examen, todas las pacientes pasaron por una consulta de consejería genética, en la cual se discutieron los métodos de diagnóstico prenatal disponibles, así como los riesgos, beneficios y limitaciones de cada uno de estos. Todos los procedimientos siguieron una sistemática convencional, con estudio ultrasonográfico previo para la comprobación de la vitalidad fetal, edad gestacional, número de fetos, inserción de la placenta y evaluación del volumen del líquido amniótico.

Bajo seguimiento ultrasonográfico directo y continuo, en $\mathrm{AP}$ se recolectó aproximadamente $1 \mathrm{ml}$ de líquido amniótico por semana gestacional con aguja 22 gauge, excepto en los primeros 19 procedimientos (aguja 20 gauge). En la BVCTA, una aguja calibre 20 gauge fue introducida por vía abdominal hasta la región del corion frondoso, una jeringuilla de $20 \mathrm{ml}$ con medio 199 (Adolfo Lutz) fue acoplada. Se procedió entonces a la aspiración de las vellosidades. Las muestras recolectadas fueron procesadas en el laboratorio de citogenética del centro de diagnóstico.

Con la finalidad de obtener informaciones referentes a datos obstétricos del parto y clínicos del recién-nacido, fueron enviados cuestionarios a las pacientes después del nacimiento de los niños. Cuando estos cuestionarios no fueron remitidos, el seguimiento de los casos fue completado por teléfono con la paciente o directamente con el obstetra. La pérdida fetal fue definida como todas las pérdidas gestacionales espontáneas ocurridas después de la realización de los exámenes. Se agruparon las pérdidas en tres categorías de acuerdo con la época en que ocurrieron: 1) hasta 30 días después del procedimiento; 2) entre 31 días y 28 semanas gestacionales; 3) por encima de 29 semanas gestacionales (natimortos y óbitos neonatales, hasta el día de vida 30).

Para determinar si había alguna diferencia estadísticamente significativa entre AP y BVCTA en cuanto a la frecuencia de pérdidas fetales y anomalías congénitas, fueron aplicadas pruebas estadísticas que consistieron en el análisis de tablas de contingencia, generalmente reducidas a las dimensiones $2 \times 2$, a las cuáles les fue aplicada la prueba exacta de Fisher. En tablas $3 \times 2$ se empleó la prueba de chi-cuadrado, seguido por la prueba de Haberman (análisis de residuos ajustados) para localizar las clases responsables por la significancia, cuando ésta tuvo lugar. En todas las pruebas se adoptó un nivel crítico de significancia estadística del $5 \%(\mathrm{a}=0,05)$. Fueron analizados también parámetros como: edad gestacional del parto (considerándose parto prematuro aquel ocurrido antes de la semana 37) y peso del recién-nacido (considerándose un peso inferior a $2.500 \mathrm{~g}$ como peso bajo al nacimiento).

\section{Resultados}

La edad y la historia obstétrica previa fueron semejantes en los dos grupos de estudio (ver tabla 1). La indicación más común para la realización de los procedimientos fue la edad materna avanzada (un $65,7 \%$ para AP y un $75,1 \%$ para BVCTA). Ver tabla 2 para otras indicaciones y tabla 3 para el número de procedimientos por edad materna y edad gestacional. 
Tabla 1. Características maternas e historia obstétrica previa.

\begin{tabular}{|l|l|l|}
\hline & \multicolumn{1}{|c|}{ AP } & \multicolumn{1}{c|}{ BVCTA } \\
\hline Media edad materna $(m \pm 1$ d.p $)$ & $35,7( \pm 4,5)$ & $36,7( \pm 5,2)$ \\
\hline Media edad gestacional $(\mathrm{m} \pm 1 \mathrm{~d} . \mathrm{p})$ & $13,7( \pm 0,7)$ & $13( \pm 0,9)$ \\
\hline Primer embarazo & $92(21,3 \%)$ & $87(20,8 \%)$ \\
\hline $\begin{array}{l}\text { Número medio de gestaciones } \\
\text { anteriores }\end{array}$ & $1,6(1-7)$ & $1,2(1-5)$ \\
\hline Aborto espontáneo previo & $128(29,6 \%)$ & $95(22,7 \%)$ \\
\hline Interrupción previa de la gestación* & $10(2,3 \%)$ & $12(2,9 \%)$ \\
\hline Localización de la placenta & \multicolumn{2}{|l}{} \\
\hline Anterior & $192(44,4 \%)$ & $228(54,5 \%)$ \\
\hline Posterior & $210(48,6 \%)$ & $147(35,2 \%)$ \\
\hline Fúndica & $21(4,9 \%)$ & $27(6,5 \%)$ \\
\hline Lateral & $9(2,1 \%)$ & $16(3,8 \%)$ \\
\hline $\begin{array}{l}\text { Procedimientos con seguimiento } \\
\text { completo por anomalía fetal }\end{array}$ & 432 & 418 \\
\hline
\end{tabular}

Fuente. Elaboración propia.

Tabla 2. Indicaciones para la realización de los procedimientos.

\begin{tabular}{|l|l|l|}
\hline \multicolumn{1}{|c|}{ Indicaciones } & \multicolumn{1}{|c|}{ AP } & \multicolumn{1}{c|}{ BVCTA } \\
\hline Edad materna avanzada $\geq 35$ años & $284(65,7 \%)$ & $314(75,1 \%)$ \\
\hline${ }^{\circ}$ Sin otras indicaciones & $240(55,5 \%)$ & $304(72.7 \%)$ \\
\hline${ }^{\circ}$ Con otras indicaciones & $44(10,2 \%)$ & $10(2,4 \%)$ \\
\hline Anomalía cromosómica previa & $6(1,4 \%)$ & $3(0,7 \%)$ \\
\hline $\begin{array}{l}\text { Hijo anterior con malformación } \\
\text { congénita }\end{array}$ & $4(0,9 \%)$ & $4(1,0 \%)$ \\
\hline $\begin{array}{l}\text { Anomalía detectada en la } \\
\text { ultrasonografía }\end{array}$ & $5(1,2 \%)$ & $1(0,2 \%)$ \\
\hline Rearreglo cromosómico en los padres & $1(0,2 \%)$ & - \\
\hline $\begin{array}{l}\text { Anomalía cromosómica en BVC } \\
\text { mosaico }\end{array}$ & $16(3,7 \%)$ & - \\
\hline $\begin{array}{l}\text { Anomalía cromosómica en BVC no } \\
\text { mosaico }\end{array}$ & $4(0,9 \%)$ & - \\
\hline Aborto a repetición & $8(1,8 \%)$ & $1(0,2 \%)$ \\
\hline Determinación del sexo fetal & - & $1(0,2 \%)$ \\
\hline Edad materna <35 ańos & $2(0,5 \%)$ & - \\
\hline Ansiedad & $94(21,8 \%)$ & $56(13,4 \%)$ \\
\hline Anomalía cromosómica previa & $13(3,0 \%)$ & $15(3,6 \%)$ \\
\hline $\begin{array}{l}\text { Hijo anterior con malformación } \\
\text { congénita }\end{array}$ & $9(2,1 \%)$ & $10(2,4 \%)$ \\
\hline Anomalía en la ultrasonografía & $7(1,6 \%)$ & $7(1,7 \%)$ \\
\hline $\begin{array}{l}\text { Anomalía cromosómica en AVC } \\
\text { mosaico }\end{array}$ & $8(1,8 \%)$ & - \\
\hline $\begin{array}{l}\text { Anomalía cromosómica en AVC no } \\
\text { mosaico }\end{array}$ & $9(2,1 \%)$ & $1(0,2 \%)$ \\
\hline Aborto a repetición & & \\
\hline
\end{tabular}

\begin{tabular}{|l|l|l|}
\hline Rearreglo cromosómico en los padres & - & $3(0,7 \%)$ \\
\hline Determinación del sexo fetal & - & $9(2,2 \%)$ \\
\hline Infección congénita & $6(1,4 \%)$ & $3(0,7 \%)$ \\
\hline
\end{tabular}

Fuente. Elaboración propia.

Tabla 3. Número de procedimientos por edad materna y edad gestacional.

\begin{tabular}{|c|c|c|c|c|c|c|c|c|}
\hline \multirow{3}{*}{ 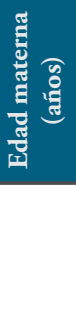 } & \multicolumn{6}{|c|}{ Semanas de gestación } & \multirow{2}{*}{\multicolumn{2}{|c|}{ Total }} \\
\hline & \multicolumn{2}{|c|}{$12-12^{6 / 7}$} & \multicolumn{2}{|c|}{$13-13^{6 / 7}$} & \multicolumn{2}{|c|}{$14-14^{6 / 7}$} & & \\
\hline & 安 & 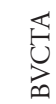 & $\dot{z}$ & 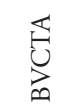 & z & 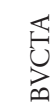 & 安 & 岕 \\
\hline$\leq 34$ & 16 & 53 & 77 & 25 & 50 & 22 & 143 & 100 \\
\hline $\begin{array}{l}35- \\
39\end{array}$ & 15 & 104 & 103 & 38 & 83 & 32 & 201 & 174 \\
\hline$\geq 40$ & 5 & 72 & 51 & 40 & 32 & 32 & 88 & 144 \\
\hline 壱 & 36 & 229 & 231 & 103 & 165 & 86 & 432 & 418 \\
\hline
\end{tabular}

Fuente. Elaboración propia.

En Ap, el porcentaje de pérdidas fetales ocurridas hasta la semana 28 de gestación fue de 2,8 \% y de 2,1\% hasta el final de la gestación incluyendo óbitos neonatales. En la BVCTA el porcentaje de pérdidas fetales ocurridas hasta la semana 28 de gestación fue de 4,3\% y de 1,0\% hasta el final de la gestación incluyendo óbitos neonatales. Aunque la frecuencia de pérdidas fetales en la BVCTA esté aumentada, ese aumento no fue significativo (prueba exacta de Fisher $P=0,756)$. Aún al comparar la frecuencia de pérdida en cada semana gestacional, no se encontraron diferencias estadísticamente significativas entre los dos procedimientos (prueba exacta de Fisher $P=0,162)$. Las diferencias observadas en la frecuencia de pérdidas fetales asociada a edad materna no fueron estadísticamente significativas en $\mathrm{AP}(\mathrm{x} 2=0,376 ; 2$ g.l.; $\mathrm{P}=0,829)$, ni en la BVCTA ( $\mathrm{x} 2=2,363 ; 2$ g.L; $\mathrm{P}=0,307)$. Interrupciones voluntarias de la gestación fueron observadas en un $3,9 \%$ de AP y un $6,5 \%$ de las BVCTA (tabla 4 ). 
Tabla 4. Pérdidas fetales ocurridas después del procedimiento.

\begin{tabular}{|c|c|c|}
\hline & $\mathbf{A P}$ & BVCTA \\
\hline & 432 & 418 \\
\hline $\begin{array}{l}\text { Perdida fetal (PF) dentro de los } 30 \\
\text { días después del procedimiento }\end{array}$ & $9(2,1 \%)$ & $12(2,9 \%)$ \\
\hline $\begin{array}{l}\text { PF después de } 30 \text { días del } \\
\text { procedimiento y hasta la semana } 28 \\
\text { de gestación }\end{array}$ & $3(0,7 \%)$ & $6(1,4 \%)$ \\
\hline Natimorto & $5(1,2 \%)$ & $2(0,5 \%)$ \\
\hline Óbito neonatal & $4(0,9 \%)$ & $2(0,5 \%)$ \\
\hline TOTAL & $21(4,9 \%)$ & $22(5,3 \%)$ \\
\hline Interrupción de la gestación & $17(3,9 \%)$ & $27(6,5 \%)$ \\
\hline \multicolumn{3}{|l|}{ ' Anormalidad cromosómica } \\
\hline trisomía 21 & 5 & 10 \\
\hline trisomía 18 & 2 & 1 \\
\hline trisomía 13 & - & 1 \\
\hline otras trisomías & 2 & 2 \\
\hline $47, \mathrm{XXX}$ & 1 & - \\
\hline $47, \mathrm{XXY}$ & 3 & 1 \\
\hline Mosaicismo & 2 & 2 \\
\hline rearreglo no equilibrado & - & 1 \\
\hline $\begin{array}{l}\text { ' Anomalía detectada en la } \\
\text { ultrasonografía }\end{array}$ & 1 & 6 \\
\hline${ }^{\circ}$ Otros & 1 & 3 \\
\hline \multicolumn{3}{|l|}{ Test exacto de Fisher $\mathbf{P}=\mathbf{0 , 7 5}$} \\
\hline
\end{tabular}

Fuente. Elaboración propia.

Los datos referidos por las pacientes y los obstetras, con relación al sangrado y la pérdida de líquido amniótico, muestran que fueron mayores en $\operatorname{AP}(2,3$ $\%$ y $5,1 \%$, respectivamente) que en BVCTA $(1,2$ $\%$ y $0,7 \%)$. Esa diferencia fue significativa en el caso de la pérdida de líquido amniótico (tabla 5) y en algunos casos se relacionó con pérdida fetal. En AP, la pérdida fetal se asoció significativamente al sangrado $(P=0,001)$ y a la pérdida de líquido amniótico $(\mathrm{P}=0,020)$. En la BVCTA, la pérdida fetal se asoció significativamente a la pérdida de líquido amniótico $(\mathrm{P}=0,009)$. No se observó diferencia significativa en la incidencia de prematuros y bajo peso al nacimiento entre AP y la BVCTA (tabla 6). La incidencia de defectos congénitos fue del 5,3\% de los nacidos vivos en AP y del 4,3\% en la BVCTA. La mayor frecuencia de problemas respiratorios en $\operatorname{AP}(2,0 \%)$ no fue significativa (prueba exacta de Fisher $\mathrm{P}=0,387$ ) en comparación con la BVCTA $(1,1 \%)$. Se registraron tres casos de anomalías músculo-esqueléticas en la BVCTA (tortícolis muscular congénito, sindactilia bilateral de los $2^{\circ}$ y $3^{\circ}$ artejo y pie equinovaro) y dos casos en $\mathrm{AP}$ (tortícolis congénito y luxación congénita de la cadera). Esa diferencia no fue estadísticamente significativa (prueba exacta de Fisher $\mathrm{P}=0,677$ ). Los problemas respiratorios y músculo-esqueléticos no estuvieron asociados con pérdida de líquido amniótico después del procedimiento.

Tabla 5. Intercurrencias pos-procedimiento.

\begin{tabular}{|l|l|l|}
\hline \multicolumn{1}{|c|}{ Intercurrencias } & \multicolumn{1}{c|}{ AP } & \multicolumn{1}{c|}{ BVCTA } \\
\hline Perdida de líquido amniótico & $22(5,1 \%)$ & $3(0,7 \%)$ \\
\hline Perdida fetal & $4(18,2 \%)$ & $2(66,6 \%)$ \\
\hline Sangrado & $10(2,3 \%)$ & $5(1,2 \%)$ \\
\hline Perdida fetal & $4(40 \%)$ & $1(20 \%)$ \\
\hline Test exacto de Fisher $\mathbf{P}=\mathbf{0 , 0 0 0 1}$ & \\
\hline Test exacto de Fisher $\mathbf{P = 0 , 2 9 8}$ & \\
\hline
\end{tabular}

Fuente. Elaboración propia.

Tabla 6. Morbilidad neonatal después del procedimiento.

\begin{tabular}{|c|c|c|}
\hline Neonatos & $\begin{array}{l}\text { AP número } \\
(\%)\end{array}$ & $\begin{array}{c}\text { BVCTA } \\
\text { número }(\%)\end{array}$ \\
\hline Nacidos vivos & $394(91,2)$ & $369(88,3)$ \\
\hline $\begin{array}{l}\text { Nacimientos a término ( } \geq 37 \\
\text { semanas) }\end{array}$ & $357(90,6) \mathrm{a}$ & $337(91,3) \mathrm{a}$ \\
\hline $\begin{array}{l}\text { Nacimientos pre-termino }(<37 \\
\text { semanas })\end{array}$ & $37(9,4)$ & $32(8,7)$ \\
\hline $\begin{array}{l}\text { Media de edad gestacional al } \\
\text { nacimiento }(\mathrm{m} \pm 1 \mathrm{~d} . \mathrm{p})\end{array}$ & $38,6 \pm 1,6$ & $38,5 \pm 1,8$ \\
\hline Peso al nacimiento $\geq 2500 \mathrm{~g}$ & $361(91,6) \mathrm{b}$ & $336(80,4) \mathrm{b}$ \\
\hline Bajo peso al nacimiento $(<2500 \mathrm{~g})$ & $33(8,4)$ & $33(7,9)$ \\
\hline $\begin{array}{l}\text { Media de peso al nacimiento }(\mathrm{m} \pm 1 \\
\text { d.p) }\end{array}$ & $3189 \pm 519 \mathrm{~g}$ & $3196 \pm 533 \mathrm{~g}$ \\
\hline \multicolumn{3}{|c|}{ Anomalías congénitas } \\
\hline Problemas respiratorios & $8(2,0) c$ & $4(1,1) \mathrm{c}$ \\
\hline Problemas musculo-esqueléticos & $2(0,5) \mathrm{d}$ & $3(0,8) \mathrm{d}$ \\
\hline Otros & $11(2,8)$ & $9(2,4)$ \\
\hline Problemas cardiacos & 3 & 3 \\
\hline Catarata congénita & 1 & - \\
\hline Fisura labiopalatina & 1 & - \\
\hline Hernia inguinal & 1 & 1 \\
\hline
\end{tabular}




\begin{tabular}{|l|l|l|}
\hline Hernia umbilical & 1 & - \\
\hline Hemangioma superciliar & 1 & 1 \\
\hline Hidrocele & 2 & 1 \\
\hline Hipospadia & 1 & - \\
\hline Dilatación pielocalicial & - & 1 \\
\hline Arteria umbilical única & - & 1 \\
\hline Atresia de íleon y microcefalia** & - & 1 \\
\hline TOTAL & $\mathbf{2 1}(5,3)$ & $\mathbf{1 6}(\mathbf{4 , 3})$ \\
\hline Admisión en la UCI neonatal & $6 *(1,5)$ & $2(0,5)$ \\
\hline${ }^{*}$ todos por problemas respiratorios & \\
\hline${ }^{* *}$ bito neonatal con 6 dias de vida & \\
\hline a X2 $=0,0057$; 1 g.l., $P=0,940$ & \\
\hline bX2 $=0,0035 ; 1$ g.l., $P=0,953$ & \\
\hline test exacto de Fisher & \\
\hline c $P=0,387$ & \\
\hline$d P=0,677$ & \\
\hline
\end{tabular}

Fuente. Elaboración propia.

\section{Discusión}

Desde que fueron introducidas en el diagnóstico prenatal, la AP y la BVC han ejercido un papel relevante en la detección de anormalidades cromosómicas en el inicio del segundo trimestre de gestación. La seguridad y precisión de estos procedimientos han sido evaluadas en varios ensayos clínicos. En una institución privada, donde las pacientes se oponen a estudios aleatorizados, uno de los medios de evaluar la seguridad del diagnóstico citogenétíco prenatal es la comparación retrospectiva de la tasa de pérdida fetal y de los resultados perinatales asociados a los diferentes procedimientos invasivos disponibles. Para efectos de consejería, esa comparación es crucial.

En la población estudiada no hubo diferencia significativa $(P=0,756)$ en la pérdida fetal asociada a cada uno de los procedimientos (tabla 4). Por lo tanto, ambos pueden ser considerados alternativas equiparables para la paciente. En los resultados encontrados por Cederholm y Axelsson (11), la frecuencia de pérdida fetal espontánea fue del 1,7\% para la BVCTA y de 6,8 \% para AP (ambos realizados entre 10 5/7 y 13 6/7 semanas de gestación), una diferencia estadísticamente significativa. En el estudio aleatorizado realizado por Nicolaides y otros (15), la tasa de pérdida fetal espontánea después de la realización de $\mathrm{AP}(5,4 \%)$ fue mayor que de la BVCTA (2,1 \%). En los procedimientos realizados entre la semana 10 y la semana 11 de gestación, esa diferencia fue considerada significativa; pero, la diferencia entre los resultados obtenidos entre la semana 12 y la semana 13 de gestación (4,0 \% para AP y 2,4\% para BVC) no fue considerada significativa. La frecuencia de pérdidas fetales encontrada en la BVCTA (5,3\%) superó aquella relatada por Chuet (16), Smidt-Jensen (17) y Brambati y otros (18) que informaron un 3,1\%, un 3,6 \% y un 4,9 $\%$, respectivamente. Por otro lado, Fortuny y otros (19), relataron una frecuencia del 5,9\%. En el caso de AP las frecuencias encontradas en algunos estudios fueron más bajas, situadas en torno a un $4 \%$ $(20,21)$, a un 3,0 \% (22-24) o un 2,5\% (25-28). Sin embargo, Schulman y otros (29) encontraron una frecuencia semejante $(4,7 \%)$; y Nagel y otros (30) relataron una frecuencia mayor $(6,2 \%)$.

Se debe considerar también la edad gestacional en que los procedimientos fueron realizados. Los resultados del presente estudio siguieren un aumento (aunque estadísticamente no significativo) en la frecuencia de pérdidas fetales en edades gestacionales más bajas, especialmente inferiores a la semana 13 . Es importante también resaltar que, antes de la semana 14, la frecuencia de pérdida basal está situada alrededor del $3 \%$ (31-34). Por lo tanto, las pérdidas gestacionales relacionadas en la casuística de este estudio pueden no ser exclusivamente atribuidas a los procedimientos diagnósticos. En el año 2004 el estudio aleatorizado de Nichd, donde se incluyeron 3.775 pacientes (1.914 BVCTA y $1.861 \mathrm{AP}$ ), reportó que el porcentaje de pérdidas fetales ocurridas hasta la semana 28 de gestación fue de 2,3 \% en AP y de 2,1 en BVCTA. Los autores consideran que estos porcentajes son relativamente bajos y pueden ser atribuidos a la experiencia de los operadores que toman la muestra y el estricto seguimiento ultrasonográfico para descartar embarazos con feto anormal. No en tanto, los autores concluyen que una 
estimación más precisa para determinar la tasa real de pérdida fetal asociada a los procedimientos invasivos, puede ser la combinación de los resultados de las pérdidas ocurridas antes de la semana 20 de gestación en pacientes que hayan presentados otras intercurrencias como ruptura prematura de membranas, pedida severa de líquido amniótico, oligohidramnios y disrupción de bandas amnióticas. Con base en esta conclusión y combinando estas dos categorías, los autores reportaron 16 pérdidas de gestación en el grupo de BVC y 27 en el grupo de AP (RR 1,74; IC del 95 \%: 0,94, 3,22; P=073). Para procedimientos realizados en la semana 13 , solamente se reportaron 10 pérdidas de gestación en el grupo de BVC y 20 en el grupo de AP (RR 2,07; IC del $95 \%$ : 0,97, 4,40; $\mathrm{P}=0,054)$. Aunque estos resultados no son estadísticamente significativos, sugieren que la tasa de pérdida fetal relacionada con el procedimiento puede ser ligeramente mayor después de la $\mathrm{AP}(31)$. En estudios más recientes, Wulff y otros (35) determinaron el riesgo de pérdida fetal asociado a pruebas invasivas de primer trimestre, incluyendo pacientes con triple screnning $(\beta-\mathrm{hCg}$, PAPP-A) y comparando los resultados con una población de pacientes no sometidas a exámenes invasivos. Los riesgos de aborto espontáneo y muerte fetal no fueron mayores en las mujeres expuestas a BVC y AP comparadas con mujeres no expuestas, por lo que concluyen que estos procedimientos no se asocian con un mayor riesgo de aborto espontáneo o muerte fetal. Estos hallazgos indican que el riesgo relacionado con el procedimiento de CVS y AC es muy bajo.

Muchos son los factores que podrían estar involucrados en las discrepancias entre los resultados encontrados en diferentes centros de diagnóstico prenatal. Las características de la población, la metodología utilizada, la edad gestacional de realización de los procedimientos y la institución donde ellos son ofrecidos pueden influir en la tasa de morbiletalidad asociada $(5-7,34,35,42)$.

En este estudio, la frecuencia de pérdidas fetales encontrada en los dos procedimientos evaluados comparativamente es superior a la de los grupos arriba mencionados. Sin embargo, fueron incluidas embarazadas con riesgo de pérdida aumentado (historia de aborto habitual e hipertensión arterial).

Otro factor a tener en cuenta es la perdida de líquido amniótico. Antes de la semana 14 de gestación, el corión y el amnio no están completamente fusionados. Durante la AP el paso de la aguja a través de un punto en el que el amnio y el corion no se fusionan puede aumentar el riesgo de una fuga posterior de líquido amniótico, lo que puede aumentar el riesgo de aborto espontáneo (36-38). En la población estudiada, la frecuencia de pérdida de líquido amniótico y sangrado después de la AP fue significativamente mayor que después de la BVCTA (tabla 5). Este incremento es un hecho relativamente frecuente en los trabajos sobre AP $(11,26,28,30,39,40-42)$. Sin embargo, conforme indica la literatura, la frecuencia de pérdida de líquido amniótico y sangrado en BVCTA es más baja que la de otros procedimientos invasivos prenatales (43). Por ejemplo, Nagel y otros autores (30) no registraron casos de pérdida de líquido amniótico ni sangrado después de la AVCTA, mientras que en AP se observó 2,6 \% de sangrado y 3,5\% de pérdida de líquido amniótico. El problema principal de estas complicaciones es cuando se asocian a pérdidas fetales. Varios estudios han descrito un incremento en la frecuencia de pérdida fetal en pacientes que reportaron pérdida de líquido amniótico después de $\mathrm{AP}(30,41,42)$. Es importante resaltar el estudio de Tara y otros (42) que relaciona líquido amniótico con presencia de sangre y perdida fetal espontánea. Los autores registran que en $10 \%$ de los casos en los que se observó pérdida fetal espontánea, el líquido amniótico extraído era de aspecto sanguinolento, esta frecuencia fue estadísticamente significativa al compararla con 3,2\% en los casos en que no se presentó pérdida fetal. Igualmente reportan la relación entre la presencia de manchado y pérdida de líquido amniótico después de la amniocentesis y la incidencia de aborto espontáneo. En el 22,2 \% de los casos con aborto espontáneo y en el 1,2\% de los casos sin aborto espontáneo, se 
produjo pérdida de líquido amniótico en los primeros diez días después de la amniocentesis. El análisis estadístico mostró que la perdida de líquido amniótico después de la amniocentesis aumenta el riesgo de aborto espontáneo.

Otra preocupación con respecto a las técnicas invasivas es la asociación con anomalías congénitas, especialmente problemas respiratorios y músculo-esqueléticos. Desde que Firth y otros (44) verificaron una asociación entre la presencia de anomalías de miembros en recién nacidos y la realización de BVCTA entre la 8 y la 9 3/7 semanas de gestación, varios trabajos se dedicaron al tema. Aunque se observaron malformaciones de miembros en fetos sometidos a la BVCTA en edades gestacionales precoces, ninguno de los trabajos encontró razones para no realizar el procedimiento después de la semana 10. El probable mecanismo por lo cual la BVC podría potencialmente llevar a malformaciones fetales incluye la ruptura vascular uterino-placentária. La BVC provocaría una lesión con vasoespasmo, o la compresión de los vasos uterinos que, subsecuentemente, resultarían en subperfusión de la circulación periférica fetal. Después de la lesión inicial, podría haber ruptura subsecuente de los vasos de paredes finas de la circulación embrionaria distal lesionada, llevando a la hipoxia, necrosis y, eventualmente, absorción de las estructuras preexistentes $(45,46)$. Por lo tanto, es necesario que la BVCTA sea realizada después de la semana 11 de gestación y por profesionales adecuadamente entrenados. En el presente estudio, la frecuencia de pie equinovaro fue mayor en $\operatorname{AP}(1,66 \%)$ que en $\operatorname{AVC}(0,48 \%)$, esta diferencia no es significativa desde el punto de vista estadístico. Nicolaides y otros (15) reportan un 4,3\% y un 3,1\% de defectos congénitos al nacimiento en grupos de AP y BVCTA (ambos entre las semanas 10 y 13), respectivamente. Sundberg y otros (40), en un estudio randomizado, encontraron un aumento significativo en la tasa de pie equinovaro en el grupo de AP cuando compararon a la BVCTA. En la conclusión de los autores, la incidencia de pie equinovaro debía ser decurrente de la prematuridad en la realización del procedimiento. En siete de los casos la colecta fue realizada antes de 86 días de gestación; adicionalmente, la caída de la presión intraamniótica durante las semanas subsecuentes al procedimiento, podría influenciar en el desarrollo de los miembros inferiores. El estudio aleatorizado de Nichd (34), reporta un aumento significativo en la tasa de pie equinovaro en el grupo de AP de 13 semanas de gestación $(0,76 \%)$ comparado con BVC de la misma edad gestacional (0,16\%). Los autores citan a Lochmilller y otros (47-52) quienes indican que en la población general el pie equinovaro ocurre con una frecuencia aproximadamente de $0,1-0,3 \%$.

Se discute también si existe mayor incidencia de dificultades respiratorias en el nacimiento de fetos cuyas madres se sometieron a procedimientos invasivos. Thompson y otros (53-58) compararon la función pulmonar en neonatos cuyas madres fueron sometidas AP o BVC. Los resultados indicaron aumento significativo en la frecuencia de recién nacidos que necesitaron asistencia respiratoria en el grupo de la BVC y una tendencia a la disminución de la capacidad pulmonar residual en ambos grupos, sugiriendo que ambos procedimientos podrían lesionar la función pulmonar. La frecuencia de anormalidades congénitas en el presente estudio (tabla 6) es mayor que la reportada por Nicolaides y otros (15). Aunque más recién nacidos del grupo de AP hayan presentado problemas respiratorios, la diferencia con relación a la BVCTA no fue estadísticamente significativa (tabla 6). Sin embargo, por ser la AP realizada en edades gestacionales precoces, la disminución brusca del volumen de líquido amniótico y la consecuente descompresión temporal de la cavidad amniótica podría, de alguna manera, interferir en la función pulmonar (12). Por eso es aconsejable minimizar el volumen de líquido amniótico recolectado, especialmente en los procedimientos realizados antes de la semana 13 de gestación.

No fueron observadas diferencias significativas en la frecuencia de anomalías músculo-esqueléticas encontradas en los procedimientos (tabla 6), las 
cuales fueron semejantes a la de la población general. Aunque existan relatos aislados correlacionando anormalidades congénitas y problemas respiratorios con los procedimientos invasivos, serían necesarios estudios randomizados y de seguimiento analizando comparativamente la incidencia de malformaciones en la población general y en recién nacidos de gestaciones sometidas a las pruebas en cuestión. Solamente así sería posible estimar los riesgos reales relacionados con los procedimientos. Complicaciones perinatales y datos del parto fueron similares en ambos procedimientos.

Mucha discusión existe aún en relación al procedimiento más adecuado de diagnóstico prenatal precoz. Los datos de esta investigación, conjuntamente con los de la literatura, indican que la amniocentesis después de 14 semanas presenta un bajo riesgo de pérdida fetal o anomalías congénitas. Con operadores expertos, puede ser ofrecido rutinariamente a las pacientes. Una amniocentesis de 13 semanas puede ser reservada para casos especiales, donde debe primar por la colecta la mínima cantidad posible de líquido amniótico. La realización de la BVCTA debe ser próxima en la semana 12 gestacional. Una clara ventaja de la BVCTA sobre la amniocentesis está relacionada con el plazo de entrega de los resultados citogenéticos. Mientras la BVCTA requiere cuatro días en media, la amniocentesis requiere aproximandamente de dos semanas.

\section{Referencias}

1. Tabor A, Madsen M, Obel E, Philip J, Bang J, Noergard-Peterson B. Randomised controlled trial of genetic amniocentesis in 4606 low-risk women. Lancet.1986; 1: 1287-1293.

2. Reece E. Amniocentese genética precoce e nos trimestres intermediários. Segurança e resultados. Clínicas Obstétricas e Ginecológicas da América do Norte. Diagnostico e Tratamento Fetal. Vol 1. Rio de Janeiro: Interlivros; 1997.

3. Eddleman K, Malone F, Sullivan L, Dukes K, Berkowitz R, Kharbutli Y, and others. Pregnancy loss rates after midtrimester amniocentesis. Obstet Gynecol. 2006; 108(5): $1067-72$.

4. Kong C, Leung T, Leung T, Chan L, Sahota D, Fung T, and others. Risk factors for procedure-related fetal losses after mid-trimester genetic amniocentesis. Prenat Diagn. 2006; 26(10): 925-30.
5. Anuwutnavin S, Chanprapaph P, Ruangvutilert P, Eammatta M, Tontisirin P. Short-term outcomes after second-trimester genetic amniocentesis in Siriraj Hospital. International Journal of Gynecology and Obstetrics. 2014; 124: 222-225. doi: 10.1016/j.ijgo.2013.09.019.

6. Connolly K, Eddleman K. Amniocentesis: A contemporary review. World J Obstet Gynecol. 2016; 5(1): 58-65.

7. Bernal L. Evaluación de la amniocentesis precoz en una institución brasileńa de diagnóstico prenatal. NOVA. 2012; 10(17): 12-24.

8. Naccache N, GollopT, Auler-Bittencourt E, Vianna-Morgante A, Eigier A. Cytogenetic analysis of first trimester chorionic villi sampling. Rev. Brasil. Genet. 1987; 10: 277-287.

9. Gollop T, Pieri P, Naccache N, Auler-Bittencourt E, Eigier A, Hauschild D. Transabdominal Chorionic Villus Sampling: experience with 70 cases. Rev. Brasil. Genet. 1990; 13(3): 591-597.

10. Gollop T, Naccache N, Campos I, Fieri P. Amostra de Vilo Corial: 1.290 casos. Rev. Brasil. Ginecol. Obstet. 1993; 15(2): 84-87.

11. Cederholm M, Axelsson O. A prospective comparative study on transabdominal chorionic villus sampling and amniocentesis performed at 10-13 weeks gestation. Prenat Diagn. 1997; 17(4): 311-317.

12. CEMAT, The Canadian Early and Mid-Trimester Amniocentesis Trial Group. Randomised trial to asses safety and fetal outcome of early and midtrimester amniocentesis. Lancet. 1998 ; 351:242-247.

13. Al Ibrahim A, Zidan M, Obaidly S, Khenyab N, Al Jenahi N, Al Mansori Z, Bolushi M, and others. Early Amniocentesis: The Resurrection. Qatar Foundation Annual Research Conference Proceedings. 2016.

14. Gollop T, Eigier A, Vianna-Morgante A, Naccache, N. Chorionic villi sampling for early prenatal genetic diagnosis. Rev. Brasil. Genet.. 1986; 9: 381-385.

15. Nicolaides K, Brizot M, Patel F, Snijders R. Comparison of Chorion Villus Sampling and Early Amniocentesis for karyotyping in 1492 singlenton preqnancies. Fetal. Diagn. Ther. 1996; 11: 9-15.

16. Chuet J, Goldberg J, Wohlferd M, Golbus M. Comparision of transcervical and transabdominal chorionic villus sampling loss rate in nine thousand from a single center. Am. J. Obstet. Gynecol. 1995; 173: 1277-1282.

17. Smidt-Jensen S, Permin M, Phillip J, Lundsteen, C, Zachary, $\mathrm{J}$, Fowler $\mathrm{S}$ et al. Randomized comparison of amniocentesis and transabdominal and transcervical chorionis villus sampling. Lancet. 1992; 340: 1237-1244.

18. Brambati B, Terzian E, Tognoni G. Randomized clinical trial of transaddominal versus transcervical chorionic villus sampling methods. Prenat. Diagn. 1991; 11: 285293.

19. Fortuny A, Borrell A, Soler A, Casals E, Costa D, Carrio A, and others. Chorionic villus sampling by forceps. Results of 1580 procedures from single centre. Prenat. Diagn. 1995; 


\section{5: $541-550$.}

20. Penso C, Sandstrom M, Garber M, Ladoulis M, Stryker J, Benacerraf, B. Early Amniocentesis: Report of 407 cases with neonatal follow-up. Obstet. Gynecol. 1990; 76: 1032-1036.

21. Sato K, Izuta M, Kojima T, Miyakawa T, Yokoo I, Takahashi $\mathrm{K}$, and others. Early amniocentesis risk and laboratory evaluation. Acta. Obstet. Gynaecol. Jpn. 1992; 44: 1285-1288.

22. Stripparo L, Buscaglia M, Longatti L, Ghisoni L, Dambrosio F, Guerneri, S, and others. Genetic amniocentesis: 505 cases performed before the sixteenth week of gestation. Prenat. Diagn. 1990; 10: 359-364.

23. Bravo R, Schulman L, Phillips O. Transplacental needle passage in early amniocentesis and pregnancy loss. Obstet. Gynecol. 1995; 86: 437-440.

24. Johnson J, Wilson R, Winsor E, Singer J, Dansereau J, Kalousek, D. The Early Amniocentesis Study: A randomized clinical trial of early amniocentesis versus midtrimester amniocentesis. Fetal. Diagn. Ther. 1996; 11: 85-93.

25. Elejalde B, de Elejalde M, Acuna J, Thelen D, Trujillo C, Karrmann M. Prospective study of amniocentesis performed between weeks 9 and 16 of gestation: Its feasibility, risks, complications and use in early genetic prenatal diaqnosis. Am. J. Med. Genet.. 1990; 35: 188-196.

26. Brumfield C, Lin S, Conner W, Cosper P, Davis R, Owen J. Pregnancy outcome following genetic amniocentesis at 11-14 versus 16 -19 weeks gestation. Obstet. Gynecol. 1996; 88(1): 114-118.

27. Eiben B, Hammans W, Hansen S, Trawicki W, Osthelder B, Stelzer A, and others. On the complication risk of Early Amniocentesis versus Standard Amniocentesis. Fetal. Diagn. Ther. 1997; 12: 140-144.

28. Daniel A, N, Kuah K, Reiha S, Malafiej P. A study of early amniocentesis for prenatal cytogenetic diagnosis. Prenat. Diagn. 1998; 18(1): 21-28.

29. Schulman L, Elias S, Simpson, J. Early amniocentesis: Complications in initial 150 cases compared to complication in initial 150 cases transabdominal chorionic villus sampling. Am. J. Human. Genet. 1991; 49: 231.

30. Nagel H, Vandenbussche F, Keirse M, Oepkes D, Oosterwijk J, Beverstock G, and others. Amniocentesis before 14 completed weeks as an alternative to transabdominal chorionic villus sampling: A controlled trial with infant follow-up. Prenat Diagn. 1998; 18: 465-475.

31. Philip J, Silver R, Wilson R, Thom E, Zachary J, Mohide P, and others. Late First-Trimester Invasive Prenatal Diagnosis: Results of an International Randomized Trial. Obstetrica and Gynecology. 2004; 103(6): 1164-117

32. Liu D, Jeavons B, Preston C, Pearson D. A prospective study of spontaneous miscarriage in intrasonically normal pregnancies and relevance to chorionic villus sampling. Pnenat. Diagn. 1987; 7: 223-226.

33. Frates M, Benson C, Doubilet M. Pregnancy outcome after a first trimester sonogram demonstrating fetal cardiac activity.
J. Ultrasound. Med. 1993; 12: 383-386.

34. Tharmaratnam S, Sadek S, Steele E, Harper M, Stewart F, Nevin J, and others. Early amniocentesis: Effect of removing a reduced volume of amniotic fluid on pregnancy outcome. Prenat. Diagn. 1998; 18:773-778.

35. Wulff C, Gerds T, Rode L, Ekelund C, Petersen O, Tabor A. Risk of fetal loss associated with invasive testing following combined first-trimester screening for Down syndrome: a national cohort of 147987 singleton pregnancies. Ultrasound Obstet Gynecol. 2016; 47: 38-44.

36. Sundberg K, Jorgensen F, Bang J. Experience with early amncentesis. J Perinat Med. 1995; 23: 149-158.

37. Tharmaratnam S, Sadek S, Steele E, Harper M, Nevin N, Dornan C. Transplacental early amniocentesis and pregnancy outcome. British Journal of Obstetrics and Gynaecology. 1998; 105(2): 228-230.

38. Zelig C, Knutzen D, Ennen C, Dolinsky B, Napolitano P. Chorionic Villus Sampling, Early Amniocentesis, and Termination of Pregnancy Without Diagnostic Testing: Comparison of Fetal Risk Following Positive Non-invasive Prenatal Testing. J Obstet Gynaecol Can. 2016; 38(5): 441-445

39. Hanson F, Tennant F, Hune S, Brookhyser K. Early amniocentesis: Outcome, risk, and technical problems at 12.8 weeksor less. Am. J. Obstet. Gynecol. 1992; 166: 1707-1711.

40. Sundberg K, Bang J, Smidt-Jensen S, Brocks V, Lundsteen C, Pamer J, and others. Randomised study of risk of fetal loss related to early amniocentesis versus chorionic villus sampling. Lancet. 1997; 350: 697-703.

41. Akolekar R, Beta J, Picciarelli G, Ogilvie C, D’Antonio F. Procedure-related risk of miscarriage following amniocentesis and chorionic villus sampling: a systematic review and meta-analysis. Ultrasound Obstet Gynecol. 2015; 45: 16-26

42. Tara F, Lotfalizadeh M, Moeindarbari S. The effect of diagnostic amniocentesis and its complications on early spontaneous abortion. Electronic Physician. 2016; 8(8): 27872792 .

43. Ghi T, Sotiriadis A, Calda P, Da Silva Costa F, Raine-Fenning N, Alfirevic Z, McGillivray G, on behalf of the International Society of Ultrasound in Obstetrics and Gynecology. ISUOG Practice Guidelines: invasive procedures for prenatal diagnosis in obstetrics. Ultrasound Obstet Gynecol. 2016; 48: 256-268.

44. Firth H, Boyd P, Chamberlain P, Mackenzie I, Lindenbaum $\mathrm{R}$, Huson S. Severe limb abnormalities after chorionic villus sampling at 56-66 days gestation. Lancet. 1991; 337: 762763.

45. Hoyme H, Jones K, Van Allen M, Saunders B, Benirschke K. Vascular pathogenesis of transverse limb reduction defects. J. Pediatr 1982; 101: 839-843.

46. Wapner, R. Amostragem do Vilo Coriónico. Clínicas Obstétricas e Ginecológicas da América do Norte. Diagnóstico e Tratamento Fetal. Reece E.A. Vol 1. Rio de Janeiro: Interlivros; 1997. 
47. Lochmiller C, Johnston D, Scott A,Risman N,Hecht T. Genetic epidemiology study of idiopathic talipes equinovarus. Am J Med Genet. 1998; 79: 90-96.

48. Bernal Luz Mery, López Greizy. Diagnóstico prénatal: retrospectiva. Nova. 2014; 12( 21 ): 23-36.

49. Márquez Gómez Marco Antonio, Gómez Díaz Graciela María. Accidente ofídico en el departamento de Sucre, Colombia. Nova. 2015; 13 ( 24 ): 39-46.

50. Pinilla B Gladys, Chavarro P Bibiana, Moreno A Natalia, Navarrete O Jeannette, Muńoz M Liliana. Determinación de los genes, 16S ADNr, polA, y TpN47, en la detección de Treponema pallidum subsp. pallidum para el diagnóstico de sífilis congénita. Nova. 2015; 13 ( 24 ): 17-25.

51. Corrales Lucia Constanza, Antolinez Romero Diana Marcela, Bohórquez Macías Johanna Azucena, Corredor Vargas Aura Marcela. Bacterias anaerobias: procesos que realizan y contribuyen a la sostenibilidad de la vida en el planeta. Nova. 2015; $13(24)$ : 55-81.

52. González Yuri Lilian. Evaluación de la percepción del riesgo ocupacional en trabajadores de una empresa del sector de la construcción en Bogotá D.C. Nova. 2015; 13( 23 ): 93-107.

53. Thompson P, Greenough A, Nicolaides K. Lung function measured by functional residual capacity in infants following first trimester amniocentesis or chorion villus sampling. Br. J. Obstet. Gynaecol. 1992; 99: 479-482.

54. Carrero Sandra Helena Suescún, HerediaMontoya Dina Paola, Bolaños Yoryany Mulato, Medellín Martín Orlando Pulido. Seroprevalencia de infección por Leptospira y factores de riesgo en estudiantes de una universidad de Colombia. Nova. 2017; $15(27$ ): 131-138.

55. Naranjo Flórez Ricardo Andrés. Avances y perspectivas en Síndrome de Asperger. Nova. 2014; 12( 21 ): 81-101.

56. Zuluaga Martha, Robledo Sebastian, Osorio-Zuluaga German A, Yathe Laura, Gonzalez Diana, Taborda Gonzalo. Metabolomics and pesticides: systematic literature review using graph theory for analysis of references. Nova. 2016; 14 ( 25 ): $121-138$.

57. Almonacid Urrego Carmen Cecilia, Camarillo Romero María del Socorro, Gil Murcia Zulay, Medina Medina Claudia Yasmin, Rebellón Marulanda Jennifer Viviana, Mendieta Zerón Hugo. Evaluación de factores de riesgo asociados a enfermedad cardiovascular en jóvenes universitarios de la Localidad Santafé en Bogotá, Colombia. Nova. 2016; 14(25): 9-17.

58. González Devia Johanna L., Monroy Romero Paola A., Almonacid Urrego Carmen C.. Homocisteína y otros factores de riesgo cardiovascular en niños de educación básica primaria del Colegio Distrital Manuel Elkin Patarroyo, Bogotá, D.C.Colombia. Estudio piloto. Nova. 2017 ; 15( 27 ): 103-117. 